\title{
Study of the Influence of the Hydration Level on the Electromechanical Behavior of Nafion Based Ionomeric Polymer-metal Composites Actuators
}

\author{
Laos Alexandre Hirano ${ }^{a *}$, Lenin Wagner Acerbi ${ }^{a, b}$, Kunitomo Kikuchic, Shigeki Tsuchitani ${ }^{c}$, \\ Carlos Henrique Scuracchio ${ }^{d}$
}

\author{
${ }^{a}$ Instituto de Ciência e Tecnologia, Universidade Federal de Alfenas - UNIFAL, \\ Rod. José Aurélio Vilela, 11999, CEP 37715-400, Poços de Caldas, MG, Brazil \\ ${ }^{b}$ Programa de Educação Tutorial, PET-Ciências, Rod. José Aurélio Vilela, 11999, \\ CEP 37715-400, Poços de Caldas, MG, Brazil \\ 'Micro Engineering Laboratory, Wakayama University, Sakaedani 930, \\ Wakayama city, Wakayama, 640-8510, Japan \\ ${ }^{d}$ Departamento de Engenharia de Materiais, Universidade Federal de São Carlos - UFSCar, \\ Rod. Washington Luís, SP-310, Km 235, CEP 13565-90, São Carlos, SP, Brazil
}

Received: November 13, 2014; Revised: October 20, 2015

\begin{abstract}
The ionomeric electroactive polymers-metal composites (IPMC) are materials that realize bending movements in response to an electrical stimulus and can be applied to the artificial muscles development. This paper investigated the influence of the hydration level on the electromechanical behavior of Nafion based IPMC. An experimental apparatus composed by a system to measure the bending force and to control the electrical stimulus and the relative humidity around the sample was developed. The main results indicated that samples with higher hydration level produce larger bending forces, but they show a more expressive back relaxation with lower time constant. A discussion about the results considering some models described in the literature conclude the paper.
\end{abstract}

Keywords: ionomeric polymer-metal composites, soft actuators, electromechanical characterization, nafion based actuators

\section{Introduction}

The ionomeric polymer-metal composites (IPMC) are smart materials that realize bending movements in response to an electrical stimulus. In spite of a low driving voltage (1-3V), a hydrated IPMC sample is able to perform smooth bending movements with large displacements ${ }^{1-3}$. These features allow the integration of lighter and smaller actuators with soft structure that performs smooth movements for the development of micro-robots, micro electromechanical systems (MEMS) and artificial muscles for small devices. A typical IPMC sample is composed by a high ionic conductivity polymer membrane with metallic electrodes plated on both faces. This paper presents an experimental setup to measure the force generated by Nafion based IPMC samples with platinum electrodes kept in environments with different relative humidity $(\mathrm{RH})$ and activated with several voltages (under $3 \mathrm{~V}$ ). The goal of this experiment is to investigate the influence of the hydration level of the polymeric phase on the electromechanical behaviour of the IPMC sample. The experimental data were fitted using a non-linear curve-fitting tool of Matlab based on least-squares approximation on a semi-empirical electromechanical model for bending movements realized by Nafion based IPMC samples. The main goal is a better understanding of the influence of the water content on the parameters of the described model.

*e-mail: laos.hirano@unifal-mg.edu.br

\subsection{Model}

Among several types of IPMC, the Nafion based ones have been widely studied due to their good chemical and thermal features. The Nafion ${ }^{\circledR}$ (Figure 1) is a graft copolymer composed of hydrophobic PTFE (Polytetrafluoroethylene) backbone with hydrophilic sulfonic-ether-perfluorated side chains. In hydrated form, these side groups agglomerate forming clusters surrounding some water molecules performing a domain with hydrophilic and ionic features which are responsible for the high ionic/solvent conductivity. The PTFE backbone form small crystallites distributed through an amorphous matrix responsible to the hydrophobic phase and have an important role in the mechanical and thermal properties of Nafion ${ }^{4-6}$.

In response to a step voltage applied on the electrodes of a hydrated Nafion based IPMC sample, the membrane realizes a fast bending movement toward the anode followed by a slow reverse movement toward the cathode ${ }^{1,2,-9}$. This reverse bending movement is usuallu called by back relaxation ${ }^{3}$. Several electromechanical models have been proposed to explain the mechanisms responsible to the movement of the IPMC. These models are based on several assumptions, including solvent/ionic diffusion ${ }^{1,10,11}$, electrical interactions between ionic species ${ }^{12-14}$ and the viscoelastic properties of the polymer phase $^{15}$. The references ${ }^{1,10,11}$ proposed an electromechanical model which relates the bending movements to the diffusion 


\section{$\left.+\mathrm{CF}_{2} \mathrm{CF}\right)\left(\mathrm{CF}_{2} \mathrm{CF}_{2}\right)_{\mathrm{n}}$ $\underset{\mathrm{CF}_{3}}{\mathrm{O} \mathrm{CF}_{2} \mathrm{CF}-\mathrm{O}-\mathrm{CF}_{2} \mathrm{CF}_{2} \mathrm{CF}_{2} \mathrm{SO}_{3}^{-} \mathrm{X}^{+}}$}

Figure 1. Structural formula of Nafion. $\mathrm{X}^{+}$is the representation of the counter cation.

of ion/solvent species activated by an external electrical stimulus. When a step voltage is applied on the electrodes, the mobile cationic species are attracted by the negatively charged electrode, migrating through the hydrophilic/ionic domains in the Nafion. These mobile cations drag some water molecules creating a water concentration gradient between the faces of the membrane. The face with the higher water concentration expands while the other contracts. The volume changes on the faces of the membrane result in a bending movement toward the anode. Another model proposed by the references ${ }^{12-14}$ associated the bending movements to the electrical interactions resulted from the redistribution of the mobile cations without significant water molecules migration. The charge redistribution creates a locally unbalanced net charge density between mobile cations and fixed anions. An associated electrostatic force creates an internal stress on the hydrophilic clusters that is transmitted to the polymer structure promoting the movements. The back relaxation is related to the back diffusion of the water molecules, cations, and/or to the memory effect of the Nafion backbone structure. Generally, the back relaxation is more expressive with samples fulfilled with smaller counter cations ${ }^{8}$.

A simple semi-empirical model based on the diffusion of solvent and ions was developed by Bar-Cohen et al. ${ }^{8}$. The model is composed by two time constants considering the fast bending movement toward the anode and the slow back relaxation toward the cathode. The curvature $k$ of the IPMC strip can be expressed by the Equation 1:

$k=V C\left[K_{2}-\frac{K_{1} \tau_{2}-K_{2} \tau_{1}}{\tau_{2}-\tau_{1}}+\frac{\tau_{2}\left(K_{1}-K_{2}\right)}{\tau_{2}-\tau_{1}}\right]$

where $V$ is the applied voltage, $C$ is the equivalent capacitance of the IPMC considering it is composed by two parallel plates, $K_{1}$ is the bending coefficient caused by the first fast cations displacement, $K_{2}$ is the coefficient for the bending effect of the charge equilibrium state, and $\tau_{1}$ and $\tau_{2}$ is the time constant related to the first bending movement and to the back relaxation.

The fluid accumulated on the cathode boundary creates an internal stress that is transmitted to polymeric matrix causing the bending movement. Considering that the deformation in the polymeric matrix is small enough to keep a Hookean regime, the force generated on the tip of the sample can be related to the curvature linearly. The Equation 1 can be reformulated to calculate the bending force on the tip of an IPMC sample:

$$
F=V\left[K_{2}^{\prime}-\frac{K_{l}^{\prime} \tau_{2}-K_{2}^{\prime} \tau_{I}}{\tau_{2}-\tau_{I}}+\frac{\tau_{2}\left(K_{l}^{\prime}-K_{2}^{\prime}\right)}{\tau_{2}-\tau_{I}}\right]
$$

where $K_{1}^{\prime}$ and $K_{2}^{\prime}$ can be approximated to the Equation 3 using the Young modulus $E$ :

$K_{i}^{\prime}=E C K_{i}$

Despite the simplicity of the model, the theoretical curve conforms the experimental data very well. However the proposed model did not include the contribution of the variations on the water content and/or the composition of the polymeric phase. This paper aimed to investigate the contribution of the variation of the water content in the sample on these parameters.

\section{Experimental}

\subsection{Sample preparation}

This work adopted a Nafion based IPMC with platinum electrodes. Nafion N-117 (1100 equivalent weight, $178 \mu \mathrm{m}$ thick) membranes are used as the control material for this study. The electrodes were plated on the polymers using impregnation-reduction method ${ }^{16,17}$. The Nafion membrane (as received) was immerged in $\mathrm{HCl} 1 \mathrm{M}$ solution, $60^{\circ} \mathrm{C}$ in order to impregnate $\mathrm{H}^{+}$ions. The next step was to soak the polymer in a solution with $\mathrm{P}^{2+}$ to be exchanged with $\mathrm{H}^{+}$and populate it with reducible cations. These ions were reduced with $\mathrm{NaBH}_{4}$ on the membrane surface forming the electrodes. In order to prepare IPMC with $\mathrm{Na}^{+}$as counter cation, the samples rinsed with deionized water were kept in $\mathrm{NaCl}$ $1,0 \mathrm{M}$ solution at room temperature during 24 hours. After, the membranes were rinsed with deionized water in order to remove the excess of the $\mathrm{NaCl}$ solution.

\subsection{Experimental setup}

Polymers with high ionic conductivity such as Nafion can easily adsorb/desorb water from the environment, modifying the morphology of both the hydrophilic and hydrophobic domain with consequences on the ionic conductivity and the Young modulus of the polymeric phase ${ }^{1,13,18}$. In order to study only the effect caused by the electrical stimulus on the electromechanical behaviour of an IPMC sample, avoiding the influence of variation on the water content, a chamber with RH controller was developed. The relative humidity was controlled activating independently two fans supplying moist and dry air respectively into the chamber. The fans were activated by relays controlled by an analogical signal generator, NI-9263 (National Instruments). The relative humidity in the chamber was monitored using a RH sensor, model HIH 4021 (Honeywell) and the data acquisition was performed by a 24 bits acquisition system, NI-9219 (National Instruments). As the sorption/desorption in Nafion is a slow process, it was adopted a simple on-off controller to keep the relative humidity practically constant in the chamber.

In the chamber, two parallel electrical terminals were installed as a clamper to hold and to apply the driving voltage on the IPMC samples. Both of them are based on titanium due to the good electrical conductivity and chemical stability. The driving voltage on the IPMC samples was controlled by the NI-9263. A buffer equipped with the OPA551 (Texas Instruments) was used to amplify the electrical current applied on the samples. One of the sample extremities was clamped between these electrical terminals in a cantilever configuration keeping one side completely fixed and the other free. The free extremity was trapped on the piston of a 250 gram-force load 
cell, model SLC-250G (Chantilon). According to Nemat-Nasser $\& \mathrm{Li}^{12}$ and Nemat-Nasser ${ }^{13}$, the IPMC sample expands when the water content in the Nafion phase increase. Considering the possible volume changes of the sample in response to the variations of the relative humidity of the environment in the chamber, the structure of the trap on the piston has a small clearance to allow sliding of the free extremity. The voltage, the electrical current and the force generated by the IPMC samples were monitored with the NI-9219. An interface to control the experimental parameters and to manage the acquire data was developed in Labview (Student version 2010). The Figure 2 shows a schema to represent the experimental setup.

\subsection{Experimental procedure}

The IPMC samples were clamped between the electrical terminals in the chamber and kept in environments with constant relative humidity and temperature $\left(25^{\circ} \mathrm{C}\right)$ during 60 minutes aiming to avoid any changes on the sample properties caused by the sorption/desorption of water during the data acquisition. It was investigated the electromechanical behaviour of samples kept in different environments, $\mathrm{RH}=40,60$ and $80 \%( \pm 2 \%)$. It was applied a step voltage of $U=0.5,1.0,1.5,2.0$ and 2.5 during a time long enough ( $100 \mathrm{~s})$ to occur the back relaxation. Voltages higher than $2.5 \mathrm{~V}$ were avoided due to the possible hydrolysis of the water and some damages to the platinum electrodes $^{8}$. The parameters $K_{1}^{\prime}, K_{2}^{\prime}, \tau_{1}$ and $\tau_{2}$ of the Equation 2 were extracted by the experimental data using a non-linear curve-fitting tool based on the Levenberg-Marquardt algorithm in MATLAB. This methodology provides a numerical solution to the problem of minimizing a function, generally nonlinear, over a space of parameters of the function. The minimization problem is solved by least squares curve fitting and nonlinear programming allowing finding local minimum.

\section{Results and Discussion}

The Table 1 lists the parameters described in the Equation 2 extracted from the electromechanical response of samples activated by $U=0.5,1.0,1.5,2.0$ and $2.5 \mathrm{~V}$ kept in environments with $\mathrm{RH}=40,60$ and $80 \%$ respectively.

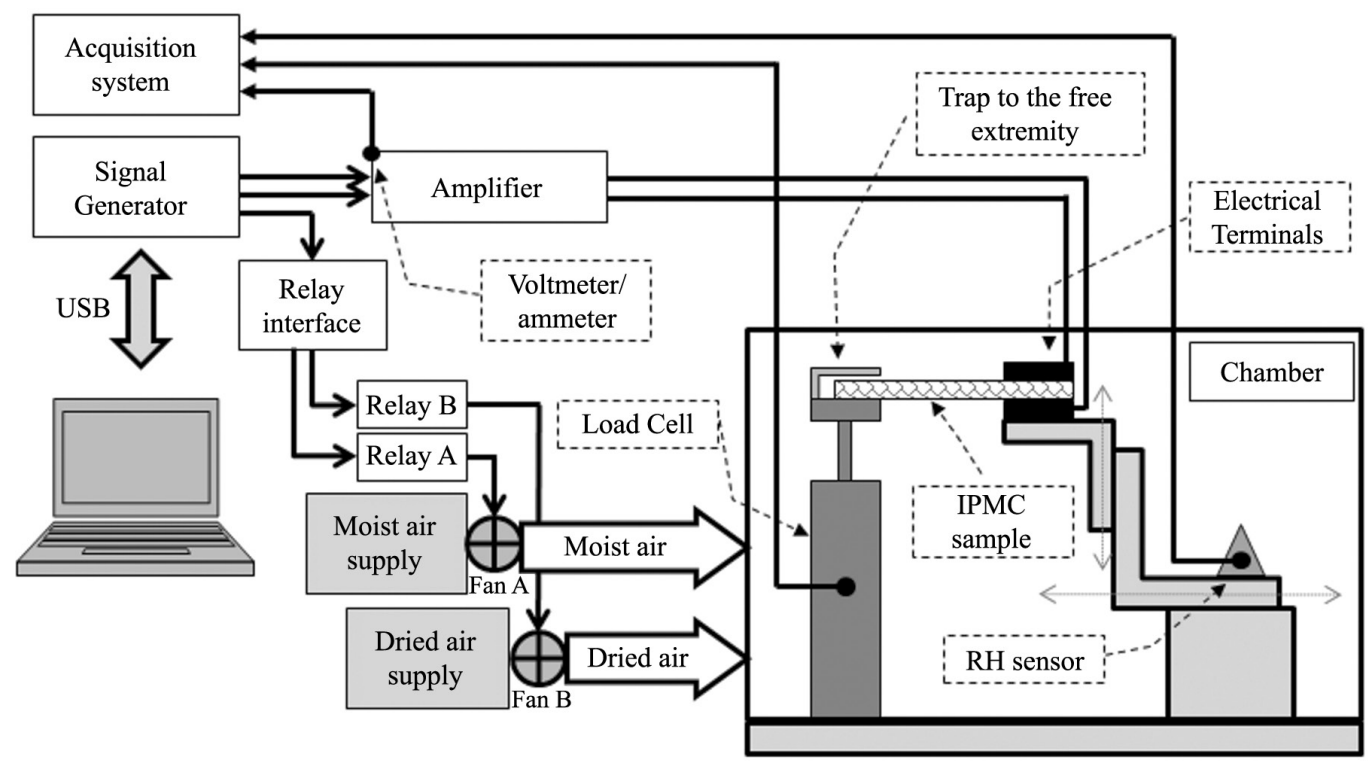

Figure 2. Experimental setup: chamber with RH controller equipped with a load cell and electrical terminals.

Table 1. Experimental parameters extracted from a N117 based IPMC with $\mathrm{Na}^{+}$.

\begin{tabular}{|c|c|c|c|c|c|c|c|}
\hline Voltage (V) & RH(\%) & $\mathbf{F}_{\max }(\mathbf{m N})$ & $\mathrm{K}_{1}^{\prime}\left(\frac{m N}{V}\right)$ & $\mathbf{K}_{2}^{\prime}\left(\frac{m N}{V}\right)$ & $\tau_{1}(s)$ & $\tau_{2}(\mathbf{s})$ & R-square \\
\hline 0.5 & & 0.031 & 0.216 & -0.078 & 4.037 & 4.059 & 0.9574 \\
\hline 1.0 & & 0.087 & 3.005 & -0.219 & 22.980 & 0.941 & 0.9979 \\
\hline 1.5 & 80 & 0.167 & 2.828 & -0.149 & 17.380 & 0.948 & 0.9971 \\
\hline 2.0 & & 0.227 & 1.883 & -0.174 & 13.440 & 1.186 & 0.9983 \\
\hline 2.5 & & 0.268 & 1.113 & -0.138 & 7.129 & 1.101 & 0.9971 \\
\hline 0.5 & & 0.036 & 3.240 & -0.306 & 76.140 & 2.276 & 0.9944 \\
\hline 1.0 & & 0.090 & 3.998 & -0.206 & 60.300 & 1.635 & 0.997 \\
\hline 1.5 & 60 & 0.115 & 6.713 & -0.182 & 72.370 & 0.958 & 0.9982 \\
\hline 2.0 & & 0.168 & 4.904 & -0.205 & 41.840 & 0.815 & 0.9992 \\
\hline 2.5 & & 0.208 & 4.720 & -0.243 & 21.200 & 0.436 & 0.9977 \\
\hline 0.5 & & 0.016 & 1.897 & -0.337 & 185.900 & 4.129 & 0.9849 \\
\hline 1.0 & & 0.059 & 4.442 & -0.264 & 208.900 & 3.479 & 0.9952 \\
\hline 1.5 & 40 & 0.098 & 4.634 & -0.178 & 204.000 & 3.481 & 0.9961 \\
\hline 2.0 & & 0.135 & 8.340 & -0.310 & 562.900 & 5.311 & 0.9927 \\
\hline 2.5 & & 0.121 & 3.574 & -0.448 & 321.730 & 6.146 & 0.999 \\
\hline
\end{tabular}




\subsection{The effect of the applied voltage}

The Figures 3 and 4 presents the electromechanical response, i.e, the blocking force variation and the current in function of the time of a Nafion based IPMC with $\mathrm{Na}^{+}$as counter cation conditioned in an environment with $\mathrm{RH}=80 \%$ submitted to a driving voltage of $U=0.5,1.0,1.5,2.0$ and $2.5 \mathrm{~V}$.

The doted and the solid curve represent the experimental results and the fitted data respectively. The R-square of all fitted curves were greater than 95\%. After an abrupt increase $\left(F_{\max }\right)$, the bending force decreased slowly until it stabilizes at a plateau. Similar patterns were presented in several references ${ }^{3,13,14,17,19}$. According to the references ${ }^{1,4,18}$, the charge transport in Nafion-like polymer are strongly associated to the ionic current which plays an important role on the bending mechanism of IPMC as discussed previously. Considering that the integral of the current as a function of time represents the amount of transported $\mathrm{Na}^{+}$in the polymeric phase, the initial current peak can be associated with a fast displacement of a significant amount of $\mathrm{Na}^{+}$resulting in an abrupt increase of the bending force resulting in a peak $\left(F_{\max }\right)$.

It was verified that the back relaxation is more expressive when a greater voltage is applied on the IPMC samples. According to some references ${ }^{12,13}$, the strong acid feature of the anionic groups of Nafion $\left(-\mathrm{SO}_{3}^{-}\right)$attracts the $\mathrm{Na}^{+}$which

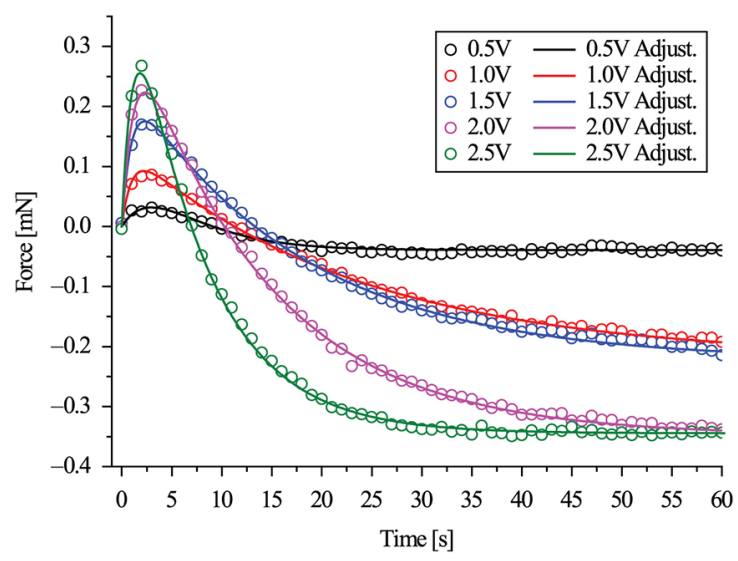

Figure 3. Electromechanical response of N117 based IPMC with $\mathrm{Na}^{+}$conditioned in an environment kept in $\mathrm{RH}=80 \%$.

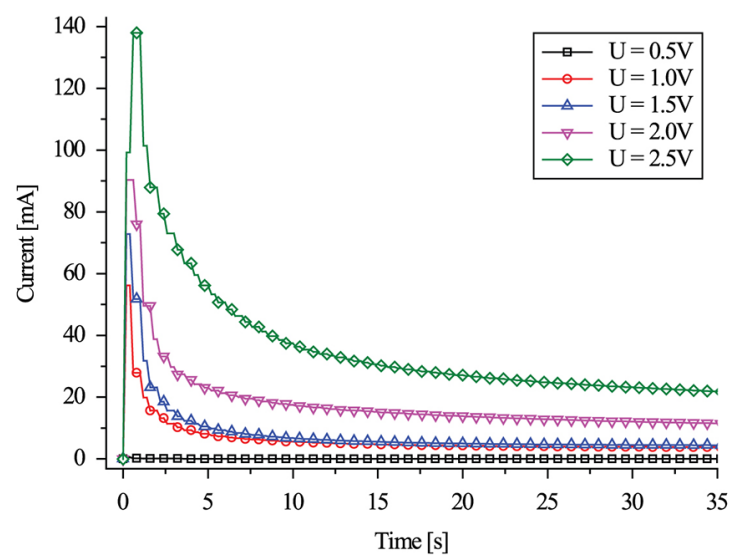

Figure 4. Electrical response of N117 based IPMC with $\mathrm{Na}^{+}$ conditioned in an environment kept in $\mathrm{RH}=80 \%$. were accumulated to the cathode boundary during the first fast bending movement. Therefore, the increase of the back relaxation and the decrease of $\tau_{2}$ can be associated to the greater amount of cations which returns to the equilibrium position as the consequence of the lack of then throughout the polymeric phase. The relation between the variation of the parameters of the Equation 2 and the applied voltage became clearer at lower hydration level.

\subsection{The effect of hydration level}

The Figures 5 and 6 presents the electromechanical response and the current in function of the time of a Nafion based IPMC with $\mathrm{Na}^{+}$as counter cation activated by a driving voltage of $U=2.0 \mathrm{~V}$ conditioned in environments with $R H=40,60$ and $80 \%$.

The increase of the water content in the IPMC samples caused an increasing of $F_{\text {max }}$ and a decreasing of $\tau_{2}, \tau_{2}$, $K_{1}^{\prime}$ and $K_{2}^{\prime}$ related to the more expressive back relaxation. Nemat-Nasser $\& \mathrm{Li}^{12}$ and Nemat-Nasser ${ }^{13}$ reported a study including the variation of Young modulus, in function of the water content, which showed that the membrane stiffness decay exponentially with the increase of the hydration level.

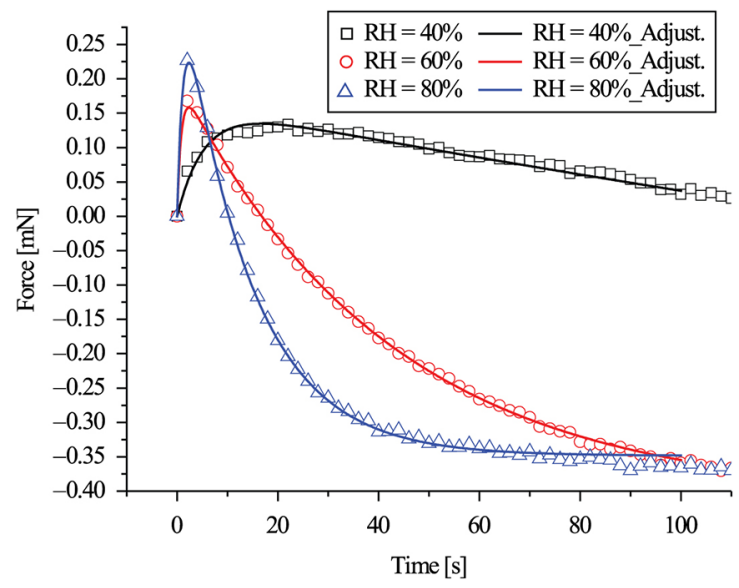

Figure 5. Electromechanical response of N117 based IPMC with $\mathrm{Na}^{+}$activated by $U=2.0 \mathrm{~V}$ conditioned in environments with different relative humidity.

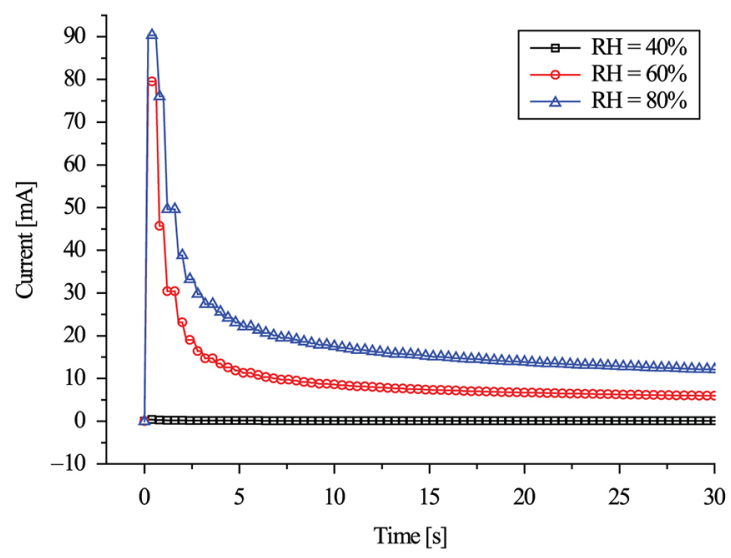

Figure 6. Electrical response of N117 based IPMC with $\mathrm{Na}^{+}$ activated by $U=2.0 \mathrm{~V}$ conditioned in environments with different relative humidity. 
The decreasing on $K_{1}^{\prime}$ and $K_{2}^{\prime}$ presented in the Table 1 can be associated to the variation of the Young modulus.

In addition, an increase of the water content in the polymeric phase causes a nonlinear increase of the ionic conductivity of a Nafion membrane, affecting directly the resistance and capacitance of the IPMC sample ${ }^{1,18}$. Therefore, in wetter conditions, the sample is softer and the Nafion phase features more water molecules allowing a greater mobility of $\mathrm{Na}^{+}$. Considering the bending mechanism discussed previously, the effect observed on the Figures 5 and 6 were expected. The relation between the variation of the parameters of the Equation 2 and the hydration level became clearer at higher voltages.

\section{Conclusions}

The effect of the hydration level on the electromechanical behaviour of $\mathrm{Na}^{+}$form Nafion based IPMC samples were investigated. In accordance to several models which describes

\section{References}

1. Hirano LA. Caracterização eletromecânica e eletro-morfológica de compósitos de polímero ionomérico e metal aplicados ao desenvolvimento de atuadores robóticos. [Thesis]. Santo André; Universidade Federal do ABC; 2013.

2. Hirano LA, Escote MT, Martins-Filho LS, Mantovani GL and Scuracchio CH. Development of artificial muscles based on electroactive ionomeric polymer-metal composites. Artificial Organs. 2011; 35(5):478-483. http://dx.doi.org/10.1111/j.15251594.2011.01259.x. PMid:21595715.

3. Bao X, Bar-Cohen Y and Lih S-S. Measurements and macromodels of ionomeric polymer-metal composites (IPMC). Smart Structures and Materials. 2002; 4695: 220-227.

4. Mauritz KA and Moore RB. State of understanding of nafion. Chemical Reviews. 2004; 104(10):4535-4585. http://dx.doi. org/10.1021/cr0207123. PMid:15669162.

5. Kreuer K, Paddison SJ, Spohr E and Schuster M. Transport in proton conductors for fuel-cell applications: simulations, elementary reactions, and phenomenology. Chemical Reviews. 2004; 104(10):4637-4678. http://dx.doi.org/10.1021/cr020715f. PMid:15669165.

6. Perles CE. Propriedades físico-químicas relacionadas ao desenvolvimento de membranas de nafion para aplicações em células a combustível do tipo PEMFC. Polímeros: Ciência e Tecnologia. 2008; 18:281-288.

7. Shahinpoor M. Ionic polymer-conductor composites as biomimetic sensors, robotic actuators and artificial muscles - a review. Electrochimica Acta. 2003; 48(14-16):2343-2353. http://dx.doi.org/10.1016/S0013-4686(03)00224-X.

8. Bar-Cohen Y, Bao X, Lih S-S, Bhattacharya K and Yu X. Characterization of the electromechanical properties of IPMC. In: Proceedings of the SPIE Smart Structures and Materials Symposium, EAPAD Conference; 2002; San Diego, EUA. San Diego: SPIE; 2002. p. 1-6.

9. Kikuchi K, Sakamoto T, Tsuchitani S and Asaka K. Comparative study of bending characteristics of ionic polymer actuators containing ionic liquids for modeling actuation. Journal of Applied Physics. 2011; 109:073505. the bending mechanism of IPMC, an increasing of the water content in the Nafion phase increased the maximum generated force and the back relaxation effect. A clear relationship between hydration level and the parameters obtained by the fitting procedure was not observed. The relation between the parameters and the hydration level became clearer at higher voltages with experiments realized at environments with lower RH.

\section{Acknowledgements}

The authors would like to thank to the Opto-Mechatronics Laboratory of Wakayama University for the samples and some parts of the experimental apparatus, the Condensed Matter Physics Laboratory of Universidade Federal do ABC for the laboratorial support, and Fundação de Amparo a Pesquisa do Estado de São Paulo - FAPESP (process 2009/09928-0) and PET-Ciências/Unifal for the financial for the financial support.

10. Kanno R, Tadokoro S, Hattori M, Takamori T, Costaftis M and Oguro K. Dynamic model of ICPF (Ionic Conducting Polymer Film) Actuator. In: Proceedings of the IEEE International Systems, Man and Cybernetics. Intelligent Systems for the 21st Century; 1995; Vancouver, Canada. Vancouver: IEEE; 1995. p. 177-182.

11. Tadokoro S, Yamagami S and Takamori T. An actuator model of ICPF for robotic applications on the basis of physicochemical hypotheses. In: Proceedings of the IEEE International Robotics and Automation Systems; 2000; San Francisco, EUA. San Francisco: IEEE; 2000. p. 1340-1346.

12. Nemat-Nasser S and Li JY. Electromechanical response of ionic polymer-metal composites. Journal of Applied Physics. 2000; 87(7):3321-3331. http://dx.doi.org/10.1063/1.372343.

13. Nemat-Nasser S. Micromechanics of actuation of ionic polymer-metal composites. Journal of Applied Physics. 2002; 92(5):2899-2915. http://dx.doi.org/10.1063/1.1495888.

14. Akle BJ, Bennett MD and Leo DJ. High-strain ionomeric ionic liquid electroactive actuators. Sensors and Actuators. A, Physical. 2006; 126(1):173-181. http://dx.doi.org/10.1016/j. sna.2005.09.006.

15. Vunder V, Punning A and Aabloo A. Mechanical interpretation of back-relaxation of ionic electroactive polymer actuators. Smart Materials and Structures. 2012; 21(11):1-9. http://dx.doi. org/10.1088/0964-1726/21/11/115023.

16. Fujiwara N, Asaka K, Nishimura Y, Oguro K and Torikai E. Preparation of gold-solid polymer electrolyte composites as electric stimuli-responsive materials. Chemistry of Materials. 2000; 12(6):1750-1754. http://dx.doi.org/10.1021/cm9907357.

17. Kikuchi $\mathrm{K}$ and Tsuchitani S. Nafion based polymer actuators with ionic liquids as solvent incorporated at room temperature. Journal of Applied Physics. 2009; 106:053519.

18. Gebel G. Structural evolution of water swollen perfluorosulfonated ionomers from dry membrane to solution. Polymer. 2000; 41(15):5829-5838. http://dx.doi.org/10.1016/S00323861(99)00770-3.

19. Tadokoro S, Fukuhara M, Maeba Y, Konyo M and Takamori T. A dynamic model of ICPF actuator considering ion-induced lateral strain for molluskan robotics. In: IEEE/RSJ International Conference on Intelligent Robots and Systems; 2002; Lausanne, Switzerland. Lausanne: IEEE; 2002. 